\title{
Integration of Microfluidic and Photonic Components within Transparent Cyclic Olefin Copolymers by Using fs Laser
}

\author{
Gian-Luca Roth ${ }^{*}$, Stefan Kefer ${ }^{1}$, Steffen Hessler ${ }^{1}$, Cemal Esen $^{2}$, and Ralf Hellmann ${ }^{1}$ \\ ${ }^{1}$ Applied Laser and Photonics Group, University of Applied Sciences Aschaffenburg, \\ 63739 Aschaffenburg, Germany \\ ${ }^{2}$ Applied Laser Technologies, Ruhr-University Bochum, 44801 Bochum, Germany \\ *Corresponding author's e-mail: gian-luca.roth@th-ab.de
}

\begin{abstract}
In this study, we report on the integration of microfluidic channels and optical components by focusing femtosecond laser radiation inside transparent cyclic olefin copolymer (COC) bulk material. An internal localized material modification is triggered based on nonlinear absorption of the laser radiation's high intensities inside the focal volume. The size and shape of the three-dimensional internal modification are controlled by using an adaptive beam shaping setup. The irradiated areas show a positive refractive index shift and can be used as Type I internal optical waveguides. Furthermore, precise control of the spatial pulse-to-pulse distance in combination with a suitable beam profile enables the integration of functional photonic elements, e.g., Bragg gratings, into the waveguide. Thus, it also enables the generation of integrated photonic sensors. In addition, internal fs laser-induced modifications are characterized by a lower thermal stability as compared to the pristine polymer material. By performing a post-annealing process step, internal hollow microstructures are created by gaseous degradation of the exposed areas. Circular microchannels can be generated in a deliberately chosen layout by employing motorized 3D stages. In comparison to etching-based fabrication methods the proposed technology facilitates unlimited channel lengths, as it omits restrictions arising from an etching selectivity and duration. Altogether, this contribution paves the way towards the fabrication of internal three-dimensional optofluidic devices, equipped with a photonic sensor. In contrast, microfluidic and photonic structures are both created by femtosecond laser direct writing inside of transparent polymers.
\end{abstract}

DOI: $10.2961 / \mathrm{jlmn} .2021 .01 .2009$

Keywords: laser material processing; polymer; ultrashort pulse; microfluidics; hollow microchannels, integrated optics, Bragg grating, optofluidics

\section{Introduction}

Lab-on-a-chip (LOC) systems are based on components whose purpose is to transport, mix, separate, and analyze small volumes of various fluids [1;2]. Several technologies have been proposed for fluid analyzation in LOCs, including electrochemical, mechanical, and optical methods [3]. Because of its high sensitivity, combined with a fast response, optical sensing principles are one of the most discussed and studied concepts for on-chip sensing [4]. Commonly, these optofluidic sensors are detecting for absorbance [5], fluorescence [6], or chemiluminescence [7] of the fluid. Furthermore, refractive index sensing can be achieved on the chip via integrated interferometers $[8 ; 9]$.

The continuous integration of these exceedingly complex functions into a single and compact chip demands multilayer systems. Since the classical production approaches originate from microsystem technology, using stacking and joining of single processed layers, the production techniques are elaborate and limited concerning multilayer structures. An uprising trend to fabricate those devices is the internal, three-dimensional processing of transparent substrates by using femtosecond laser pulses and novel direct writing techniques [10].

Based on the rapid development of femtosecond lasers in recent years, several attempts of combined internal structuring and direct writing of photonic elements have been demonstrated [11]. Standard technologies for internal structuring of glass include selective laser etching [12] and liquid supported backside ablation [13]. Integrated optical elements can be created either by a positive refractive index shift inside a laser-induced modification [14], by the creation of optical stress in-between modified tracks [15] or by generating a depressed cladding [16].

Furthermore, fluorescence excitation within the junction of a microfluidic channel and a waveguide has been demonstrated [17; 18]. In addition to this, monolithic optofluidic refractive index sensors in glass have already been developed including Fabry-Pérot interferometers [19], MachZehnder interferometers [20] and Bragg gratings [21; 22]. Transparent polymer materials are an uprising LOC substrate material class. They gain respectable interest because of low material costs (approximately $1-10 \%$ as compared to glass) adjustable physical properties and biocompatibility [23]. Especially cyclic olefin copolymers (COCs), offering a low water absorption of less than $0.01 \%$, a high glass transition temperature, and a high resistance against common solvents are highly suitable for a wide range of sensor applications [24-26]. However, the monolithic integration of polymer optofluidic devices using an ultrashort pulsed laser is still under research [27]. Internal microfluidic channels can 


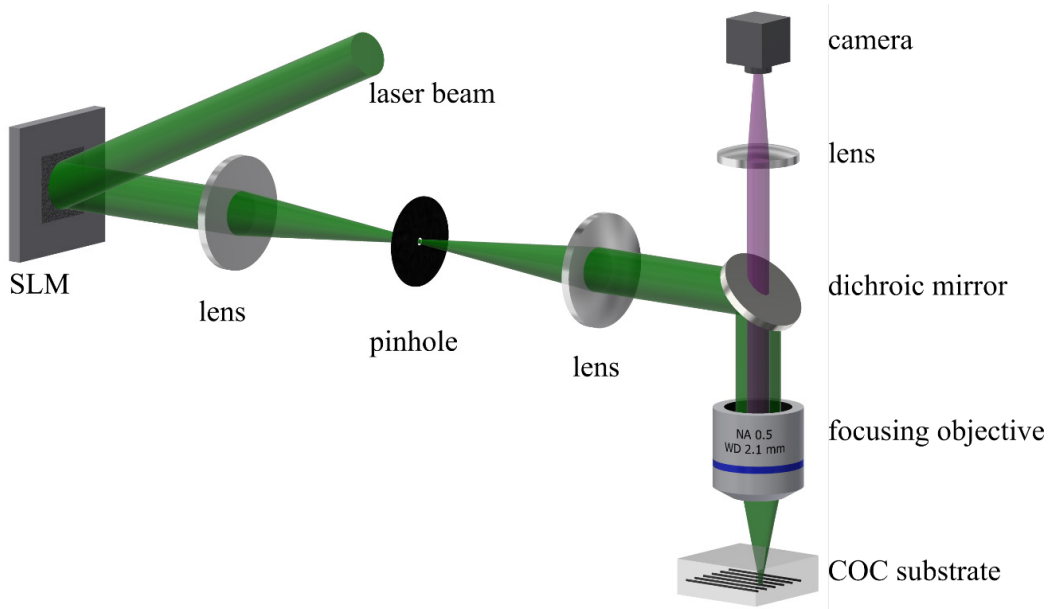

Fig. 1 Schematic illustration of the experimental setup for the generation of internal structures inside COC substrate using femtosecond laser radiation. Beam shaping is performed with the usage of a spatial light modulator (SLM) and a $4 \mathrm{f}$ telescope with an additional pinhole in front of the focusing objective to block the zeroth diffraction order.

be created by thermal decomposition of material previously modified with femtosecond laser in PMMA [28; 29] and COC [30]. Femtosecond laser direct writing of different types of waveguides has also been demonstrated in both materials [31-35]. A combined generation of both structures has not been reported so far. In this study, we report on the implementation of combined manufacturing of internal microfluidic channels and integrated optical elements in $\mathrm{COC}$ by employing only one laser processing setup.

\section{Experimental}

All structures are fabricated by employing an ultrashort pulsed laser (Light Conversion, Pharos-10-600) with a fundamental wavelength of $1028 \mathrm{~nm}$, featuring adjustable pulse duration from 220 fs to $15 \mathrm{ps}$ and variable repetition rates up to $610 \mathrm{kHz}$. By using a second harmonic generation module, a laser wavelength of $514 \mathrm{~nm}$ can be emitted. The linear polarized laser beam is shaped using a spatial light modulator (SLM; Holoeye, Pluto VIS 21) with a resolution of $1920 \times 1080$ pixels and a pixel size of $8 \mu \mathrm{m}$. A $4 \mathrm{f}$ setup $(\mathrm{f}=300 \mathrm{~mm})$ is used to image the SLM patterns at the focusing objective. To block the zeroth order of a blazed grating additionally displayed on the SLM, a pinhole is inserted at the focal plane of the telescope, as depicted in Fig. 1. The first diffracted order passes the pinhole unaffected and is focused by an air objective with an NA of 0.5 (Zeiss, EC Epiplan-Neofluar) into the material. The phase function displayed on the SLM is calculated to correct the spherical aberration lens effect caused by the refractive index mismatch between air and processed transparent polymer [36]. Beam measurements on the resulting volume inside the focus (voxel) are performed using a coaxial CCD camera (Imagingsource, DMK 27AUJ003). During processing, samples are positioned using linear translation stages with a spatial resolution of $1 \mathrm{~nm}$ (Aerotech, ANT130-XY-Plus). The focal position is controlled by a nanopositioning vertical stage with a spatial resolution of $1 \mathrm{~nm}$ (Aerotech, ANT95-50-LPlus). An overview of the process parameters used for the generation of microchannels, waveguides and Bragg gratings is given in Table 1.
Before processing, injection molded bulk $\mathrm{COC}$ material (TOPAS 6017, TOPAS Advanced Polymers) samples were cut to a size of $44 \times 22 \mathrm{~mm}$ using an ultrashort pulse laser in combination with a precision cutting head (FineCutter, Precitec). By employing a laser output power of $8.5 \mathrm{~W}$ at a pulse repetition rate of $250 \mathrm{kHz}$ and a laser wavelength of $1028 \mathrm{~nm}$ a cutting speed of $1 \mathrm{~mm} / \mathrm{s}$ is achieved. The cutting process is supported by a coaxial nitrogen stream with a pressure of 6 bar, which enables burr-free surfaces. The bulk material is characterized by a glass transition temperature of $170{ }^{\circ} \mathrm{C}$ and a tensile strength of $58 \mathrm{MPa}$. The plates had a thickness of $1.5 \mathrm{~mm}$ and a surface roughness $R_{a}$ of $25 \mathrm{~nm}$. The refractive index $\mathrm{n}$ of the material at the laser wavelength of $514 \mathrm{~nm}$ is 1.536 at room temperature calculated by Sellmeier equation.

The laser generation of microchannels consists of two process steps. Firstly, COC samples are irradiated by focused femtosecond laser pulses $(514 \mathrm{~nm})$, triggering a nonlinear absorption inside the focal volume. Arbitrary 3D geometries can be created by translating the focal spot in three dimensions inside the bulk polymer. Secondly, COC specimens are annealed between two glass substrates in a convector oven at $220^{\circ} \mathrm{C}$ for $20 \mathrm{~min}$.

Table 1 Overview of the applied laser processing parameters.

\begin{tabular}{cccc}
\hline Parameter & $\begin{array}{c}\text { Micro- } \\
\text { channel }\end{array}$ & Waveguide & $\begin{array}{c}\text { Bragg } \\
\text { grating }\end{array}$ \\
\hline Wavelength & $514 \mathrm{~nm}$ & $514 \mathrm{~nm}$ & $514 \mathrm{~nm}$ \\
Pulse duration & $450 \mathrm{fs}$ & $450 \mathrm{fs}$ & $450 \mathrm{fs}$ \\
Repetition rate & $500 \mathrm{kHz}$ & $25 \mathrm{kHz}$ & $0.5 \mathrm{kHz}$ \\
Pulse energy & $70 \mathrm{~nJ}$ & $330 \mathrm{~nJ}$ & $355 \mathrm{~nJ}$ \\
Pulse separation & $200 \mathrm{~nm}$ & $600 \mathrm{~nm}$ & $1040 \mathrm{~nm}$ \\
Beam profile & Circular & Elliptical & Elliptical
\end{tabular}

In order to characterize microchannel cross-sections, the treated specimens are ground down to the created structures 


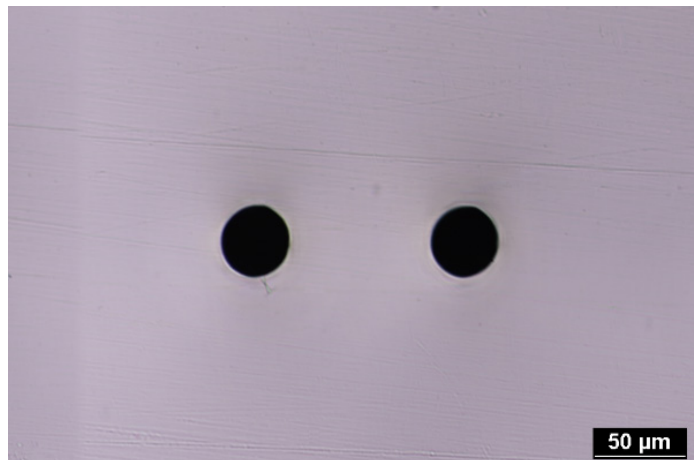

Fig. 2 Transmission microscope image of circular cross-sections of internal microchannels in COC substrate generated in a depth of $500 \mu \mathrm{m}$ using a writing speed of $100 \mathrm{~mm} / \mathrm{s}$.

and polished. Afterwards, an ultrasonic bath is used to remove debris from this process step. Channel geometries are measured by using reflection and transmission light microscopes (Nikon, 70 Eclipse LVDIA-N; Leica, DVM6).

Output profiles of the internal area of the waveguides are analyzed by using a $5 x$ microscope objective with an NA of 0.25 in combination with a CCD camera (IDS, UI1490SE-M-GL) at a wavelength of $633 \mathrm{~nm}$. Fabricated Bragg gratings are characterized by recording the reflected spectra using a source and detector interrogation system (Micron Optics, si155 HYPERION), which operates in a wavelength range from 1460 to $1620 \mathrm{~nm}$. Thereby, the spectral position of the reflected Bragg wavelength, the fullwidth at half-maximum bandwidth (FWHM), and the reflected power are examined. For temperature measurements, an internal waveguide Bragg grating is butt-coupled to a single-mode-fiber using UV curable glue (AddisionClear Wave Coatings Inc., NOA 76) and placed onto a hotplate. An additional fiber temperature sensor (OpSens) with an accuracy of $\pm 0.1^{\circ} \mathrm{C}$ was applied to the surface of the COC sample to guarantee accurate temperature readings.

\section{Results and Discussion}

\subsection{Generation of internal microfluidic channels}

The generation of microchannels in COC is performed by using a laser pulse repetition rate of $500 \mathrm{kHz}$, in combination with a pulse to pulse distance of $0.2 \mu \mathrm{m}$, resulting in a processing speed of $100 \mathrm{~mm} / \mathrm{s}$. This parameter combination induces a well controllable heat accumulation, which is necessary to generate suitable internal modifications in COC for the creation of microchannels. Processing the samples without heat accumulation, i.e., by employing a reduced laser pulse repetition rate of $5 \mathrm{kHz}$, results in not decomposable, strong scattering dark lines. As these modifications are induced by heat accumulation during processing, the resulting channel cross-section is not determined by the shape of the laser focal voxel, but defined by the resulting heat distribution during processing. This is in contrast to previous studies in PMMA, with the channel cross-section being controllable by the intensity distribution inside the focal voxel [28; 37]. In Fig. 2, typical circular microchannel cross-sections are exemplified. Their size can be controlled either by the writing speed or by the laser pulse energy, as both parameters affect heat accumulation during processing [30]. Inside the process window to generate internal microchannels in COC, only a slight influence on the microchannel's

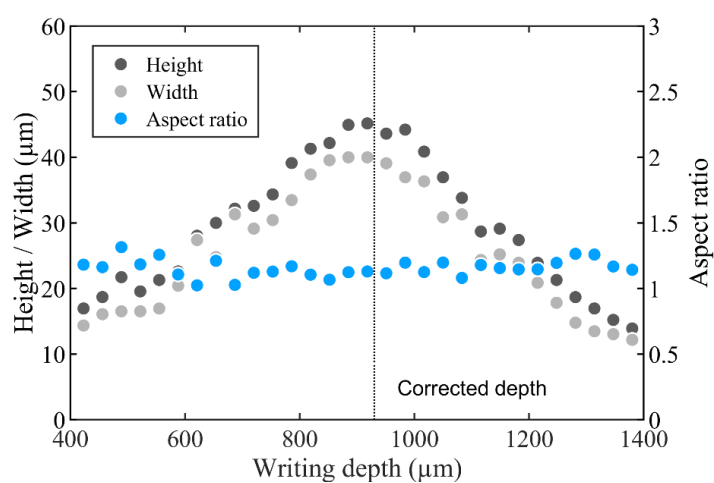

Fig. 3 Size distribution of internal microchannels written in different depths using an aberration correction for a depth of $950 \mu \mathrm{m}$.

aspect ratio of approximately one is determined by both process parameters. The profile surface roughness along a line in the orientation of an internal microchannel is determined to be below $\mathrm{R}_{\mathrm{a}} 500 \mathrm{~nm}$.

The results to pre-compensate the spherical aberration lens effect, performed with the SLM, are demonstrated in Fig. 3. At a writing speed of $100 \mathrm{~mm} / \mathrm{s}$ and a laser pulse energy of $70 \mathrm{~nJ}$, microchannels are generated in different depths of the polymer substrate. The SLM based pre-compensation phase function corresponds to a writing depth of $950 \mu \mathrm{m}$. Due to the positive and negative spherical aberration lens effects above and below the compensation depth, the intensity of the laser radiation inside the voxel is reduced, resulting in significantly smaller channel sizes. The aspect ratio of the microchannels is constant around one due to the heat-induced origin of the modification. In contrast to this, spherical aberration effects result in an elongation of the channel height at the generation of internal structures in PMMA [28].

In addition, at writing depths which are not corresponding to the predefined compensation, second and third modifications below the initial focal zone are created. Those undesired structures disturb the circular channel cross-sectional shape and need to be prevented by employing a suitable spherical aberration correction.

\subsection{Generation of integrated optical elements}

The creation of integrated optical elements in transparent polymers is based on single-shot induced refractive index
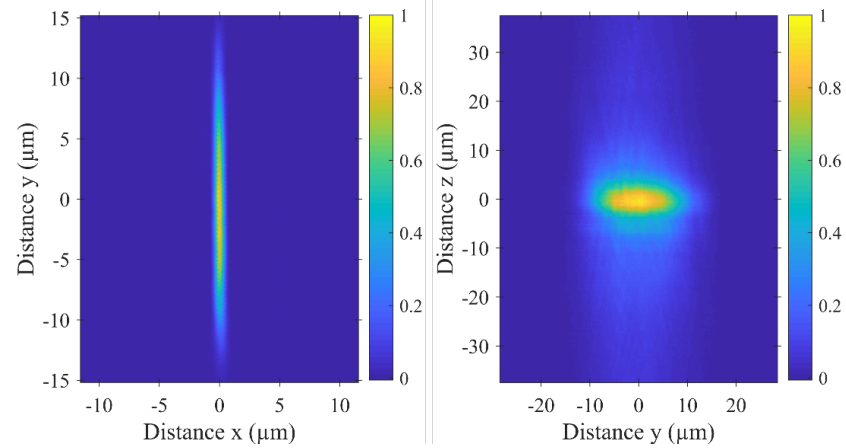

Fig. 4 Result of the measured normalized beam profile of the elliptical focal voxel in $x-y$ cross-section (left) at the focal position and $y-z$ cross-section (right) using an ellipticity of 15 and an effective NA of 0.24 on the major axis. 


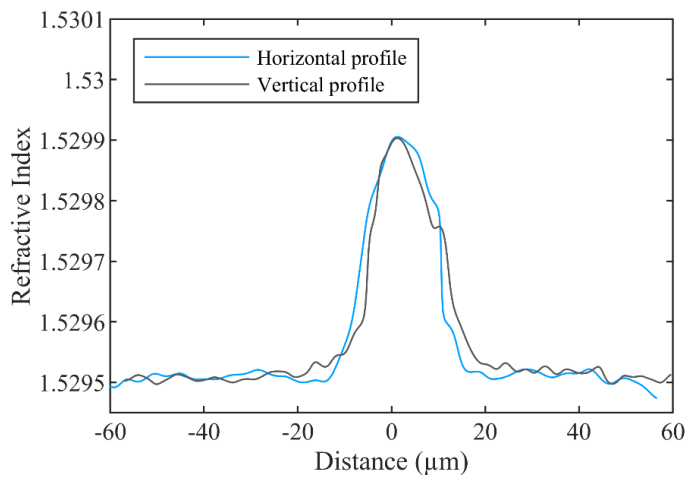

Fig. 5 Horizontal and vertical cross-section of a positive refractive index modification inscribed using a laser pulse energy of $336 \mathrm{~nJ}$ and measured at a wavelength of $633 \mathrm{~nm}$.

modifications. Compared to the generation of internal microfluidic structures, this process is not based on heat accumulation but on a single shot photo-induced modification and is performed at a laser pulse repetition rate of $25 \mathrm{kHz}$. The size and shape of the internal modifications are determined by the intensity distribution of the focal voxel shown in Fig. 4, but are also depending on laser process parameters such as laser power or pulses per point. The generation of an upright standing thin disk energy distribution is performed by an additional virtual slit mask which is inserted on the phase function correcting for spherical aberration displayed on the SLM [37; 38]. The resulting shape of the focal voxel is characterized by a horizontal size of $1.5 \mu \mathrm{m}$ in $\mathrm{x}$-direction, $25 \mu \mathrm{m}$ in $\mathrm{y}$-direction (both measured at $1 / \mathrm{e}^{2}$ ), and a focal height of $7.5 \mu \mathrm{m}$ (FWHM). The pulse-to-pulse distance is set to $600 \mathrm{~nm}$, which corresponds to a writing speed of $15 \mathrm{~mm} / \mathrm{s}$.

The generated structures exhibit a maximum positive refractive index shift of $4 \cdot 10^{-4}$ RIU [35] measured at a wavelength of $633 \mathrm{~nm}$ and are consequently appropriate to be used as Type I waveguides [39; 40]. Vertical and horizontal cross sections of a refractive index modification inscribed using a laser pulse energy of $336 \mathrm{~nJ}$ are given in Fig. 5. The refractive index is measured by using a Mach-Zehnder interferometer [41]. The output profiles of waveguides at a wavelength of $633 \mathrm{~nm}$ inscribed with different laser pulse energies, are given in Fig. 6. By using a laser pulse energy of $330 \mathrm{~nJ}$, the size and shape of the output profile of the integrated waveguide correspond well with the intensity distribution of the focal voxel depicted in Fig. 4. At a higher laser pulse energy of $375 \mathrm{~nJ}$, internal damage is induced along with the modification, which disturbs the optical waveguide. At a lower laser pulse energy of $260 \mathrm{~nJ}$, the positive refractive index shift is insufficient to perform as a well-defined Type I waveguide. The propagation loss of fs laser written waveguides in COC using comparable processing parameters has been previously reported by the authors to be $3.2 \mathrm{~dB} / \mathrm{cm}$ in a wavelength range from 1545 to $1555 \mathrm{~nm}$ [35].

In addition to that, femtosecond laser direct writing enables the generation of functional sensor elements, such as Bragg gratings, inside of the transparent polymer substrate. A mask-less point-by-point writing approach, is applied to inscribe a periodic refractive index modulation. A single laser pulse induces each period of the Bragg grating. The voxel defines the lateral grating width. Due to the elliptical profile of the focal voxel (cf. Fig. 4), the period of the grating can be selected to be significant smaller as its width. The optical connection between a waveguide and Bragg grating is depicted in Fig. 7 (left). Both structures are written as independent lines. Thus, the transition between both endpoints is a critical factor for the device performance. On the one hand, a gap between both components results in an unguided region with increased optical loss. On the other hand, an overlap of grating and waveguide results in damage along the double irradiated zone. At optimized layout conditions, we estimate a coupling loss of about $3 \%$ based on simulations (Synopsys, RSoft), which include the profile of the refractive index modification shown in Fig 5. and the effective refractive index of the Bragg Grating given in Fig. 7 (middle). In this study, waveguide-coupled Bragg gratings are inscribed with a period of $1040 \mathrm{~nm}$ at a laser pulse repetition rate of $500 \mathrm{~Hz}$ using a laser pulse energy of $355 \mathrm{~nJ}$ in a depth of $500 \mu \mathrm{m}$ below the surface. The fabricated gratings show a characteristic Bragg wavelength $\lambda_{B}$ of $1581.8 \mathrm{~nm}$, which corresponds well with its spatial period $\Lambda$ in accordance with Eq. (1):

$$
m \cdot \lambda_{B}=2 \cdot n_{e f f} \cdot \Lambda
$$

with $m$ being the order of the reflection and $n_{\text {eff }}$ representing the effective refractive index. The reflection spectrum of an internal waveguide coupled Bragg grating with a length of $15 \mathrm{~mm}$ is depicted in Fig. 7 (middle). It exhibits a Bragg reflection at $1581.8 \mathrm{~nm}$ and an FWHM of $152 \mathrm{pm}$. The spectral position of an internal waveguide coupled Bragg grating is sensitive to changes of the effective refractive index and the spatial period of the grating. For any sensing application, e.g., temperature or strain, the reduction of
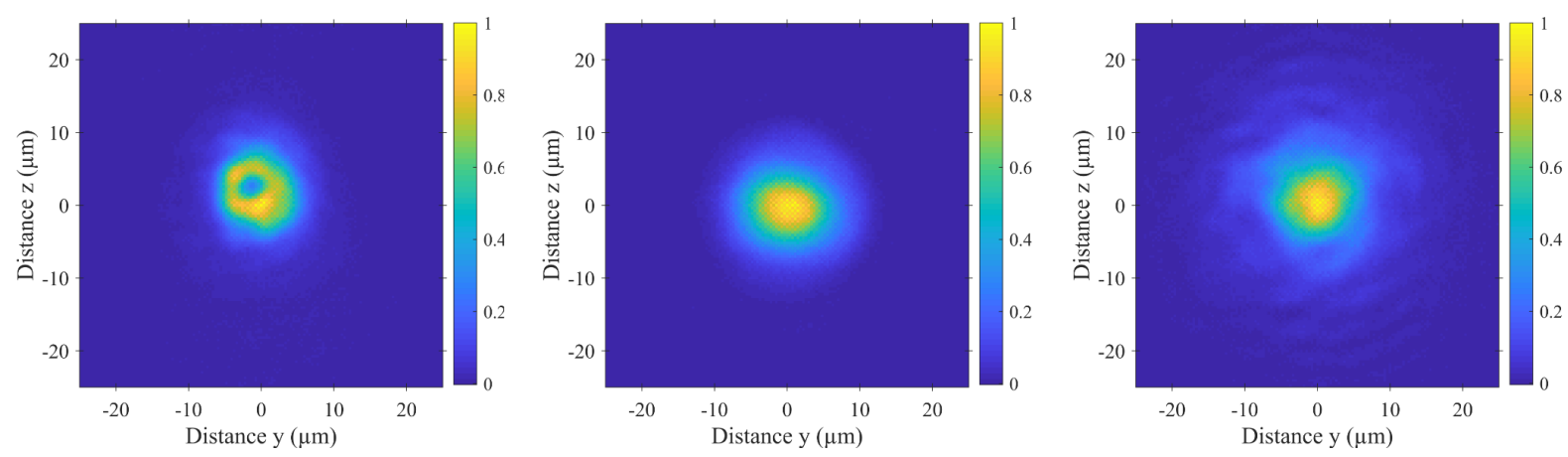

Fig. 6 Result of the measured normalized intensity distribution at the output of an internal waveguide at a wavelength of $633 \mathrm{~nm}$. The photonic structures are fabricated using laser pulse energies of $375 \mathrm{~nJ}$ (left), $330 \mathrm{~nJ}$ (middle), and $260 \mathrm{~nJ}$ (right), respectively. 

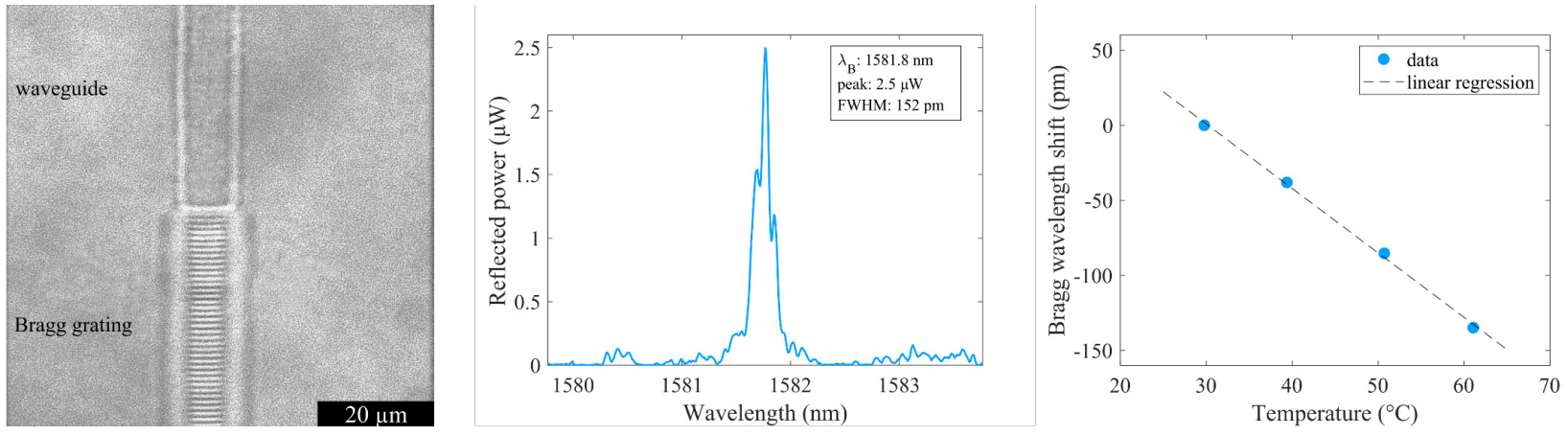

Fig. 7 Transmission microscope image of the connection between femtosecond laser written waveguide (top) and point-by-point inscribed Bragg grating (bottom, left). The reflection spectrum of a waveguide coupled optical grating with a spatial period of $1040 \mathrm{~nm}$ is illustrated in the middle. The spectral shift of the Bragg wavelength over temperature is demonstrated in the right figure.

potential cross sensitivities is of most importance. In this respect, the low water absorption is a major advantage of COCs compared to other polymer materials like PMMA. It also enables a direct application as an integrated temperature sensor. A temperature increase of the COC-based Bragg grating leads to a negative refractive index change [42] and a simultaneous increase in the grating period. The overall temperature sensitivity of the generated waveguide-coupled Bragg grating is determined as $-4.3 \mathrm{pm} / \mathrm{K}$, as depicted in Fig. 7 (right). According to the authors' preliminary studies, this value is comparable to diffused Bragg gratings generated within COCs employing an adapted phase mask procedure [25].

\section{Conclusion}

In this contribution, the femtosecond laser generation of internal microfluidic and optical structures in transparent COC substrates are studied. Microfluidic channels are created in a two-step process consisting of an internal laser-induced modification based on heat accumulation during exposure and subsequent annealing. The circular cross-sectional shape of the internal microchannels is defined by the heataffected zone. By using an adaptive beam shaping device, microchannels can be created inside of the transparent material, whereas their aspect ratio is independent of the writing depth. Integrated microoptical components, i.e., waveguides or waveguide coupled Bragg gratings, can be inscribed into the COC substrate with the shape of the modified refractive index being defined by the intensity distribution of the voxel. In addition, the shape of the voxel can be precisely controlled. The applied point-by-point inscription technology enables the creation of Bragg gratings of almost any desired period by controlling the distance between the modifications. Furthermore, the application of a waveguide coupled Bragg grating as an internal temperature sensor is demonstrated. In summary, the demonstrated femtosecond laser-based fabrication methods, performed on a single laser processing setup, enable the straightforward and flexible generation of microfluidic and photonic sensing structures within a bulk polymer. Thus, the proposed technology constitutes a foundation for the advanced fabrication of polymer LOC devices.

\section{References}

[1] E. K. Sackmann, A. L. Fulton, and D. J. Beebe: Nature, 507, (2014) 181.
[2] E. Samiei, M. Tabrizian, and M. Hoorfar: Lab Chip, 16, (2016) 2376.

[3] N. M. M. Pires, T. Dong, U. Hanke, and N. Hoivik: Sensors, 14, (2014) 15458.

[4] C. Monat, P. Domachuk , and B. J. Eggleton: Nat. Photonics, 1, (2007) 106.

[5] B. G. Splawn and F. E. Lytle: Anal. Bioanal. Chem., 373, (2002) 519.

[6] R. Mazurczyk, J. Vieillard, A. Bouchard, B. Hannes, and S. Krawczyk: Sens. Actuator B Chem., 118, (2006) 11. [7] B. Al Mughairy and H. A. Al-Lawati: TrAC Trends in Analytical Chemistry, 124, (2020) 115802.

[8] B. J. Luff, J. S. Wilkinson, J. Piehler, U. Hollenbach, J. Ingenhoff, and N. Fabricius: J. Light. Technol., 16, (1998) 583.

[9] M. I. Lapsley, I.-K. Chiang, Y. B. Zheng, X. Ding, X. Mao, and T. J. Huang: Lab Chip, 11, (2011) 1795.

[10] F. Sima, K. Sugioka, R. M. Vázquez, R. Osellame, L. Kelemen, and P. Ormos: Nanophotonics, 7, (2018) 613.

[11] J. M. Maia, V. A. Amorim, D. Alexandre, and P. V. S. Marques: "Advances in Fs-Laser Micromachining: Towards the Development of Optofluidic Devices" in "Optics, photonics and laser technology" ed. by P. Ribeiro, D. L. Andrews, and M. Raposo, (Springer, Series in Optical Sciences 222, 2017) p. 119.

[12] J. Gottmann, M. Hermans, N. Repiev, and J. Ortmann: Micromachines, 8, (2017) 110.

[13] Y. Li and S. Qu: Curr. Appl. Phys., 13, (2013) 1292.

[14] M. Ams, G. D. Marshall, D. J. Spence, and M. J. Withford: Opt Express, 13, (2005) 5676.

[15] J. Burghoff, S. Nolte, and A. Tünnermann: Appl. Phys. A, 89, (2007) 127.

[16] Q. Zhang, D. Yang, J. Qi, Y. Cheng, Q. Gong, and Y. Li: Opt Express, 25, (2017) 13263.

[17] R. Martinez Vazquez, R. Osellame, M. Cretich, M. Chiari, C. Dongre, H. J. W. M. Hoekstra, M. Pollnau, H. van den Vlekkert, R. Ramponi, and G. Cerullo: Anal. Bioanal. Chem., 393, (2009) 1209.

[18] A. Crespi, R. Osellame, and F. Bragheri: Opt. Mater. X, 4, (2019) 100042.

[19] J. M. Maia, V. A. Amorim, D. Viveiros, and P. V. S. Marques: J. Phys. Photonics, 2, (2020) 15003.

[20] A. Crespi, Y. Gu, B. Ngamsom, H. J. Hoekstra, C. Dongre, M. Pollnau, R. Ramponi, H. H. van den Vlekkert, P. Watts, and G. Cerullo: Lab Chip, 10, (2010) 1167.

[21] V. Maselli, J. R. Grenier, S. Ho, and P. R. Herman: Opt Express, 17, (2009) 11719. 
[22] J. M. Maia, V. A. Amorim, D. Alexandre, and P. V. S. Marques: J. Light. Technol., 35, (2017) 2291.

[23] P. Abgrall and A. M. Gue: J. Micromech. Microeng., 17, (2007) R15.

[24] P. S. Nunes, P. D. Ohlsson, O. Ordeig, and J. P. Kutter: Microfluid. Nanofluid., 9, (2010) 145.

[25] M. Rosenberger, G.-L. Roth, B. Adelmann, B.

Schmauss, and R. Hellmann: IEEE Photonic. Tech. L., 29, (2017) 885.

[26] M. Rosenberger, M. Girschikofsky, M. Förthner, S.

Belle, M. Rommel, L. Frey, B. Schmauss, and R.

Hellmann: J. Opt, 20, (2017) 01LT02.

[27] S. M. Eaton, C. D. Marco, R. Martinez-Vazquez, R. Ramponi, S. Turri, G. Cerullo, and R. Osellame: J. Biophotonics, 5, (2012) 687.

[28] G.-L. Roth, S. Rung, C. Esen, and R. Hellmann: Opt Express, 28, (2020) 5801.

[29] G.-L. Roth, C. Esen, and R. Hellmann: Opt Express, 25, (2017) 18442.

[30] G.-L. Roth, C. Esen, and R. Hellmann: Proc. SPIE. Vol. 11268, (2020) 112681L.

[31] W.-H. Yuan, J.-M. Lv, C. Cheng, X.-T. Hao, and F. Chen: Opt. Mater., 49, (2015) 110.

[32] W. M. Pätzold, A. Demircan, and U. Morgner: Opt Express, 25, (2017) 263.

[33] W. Watanabe, S. Sowa, T. Tamaki, K. Itoh, and J. Nishii: Jpn. J. Appl. Phys., 45, (2006) L765.

[34] P. J. Scully, A. Baum, D. Liu, and W. Perrie: "Refractive Index Structures in Polymers" in "Femtosecond Laser Micromachining: Photonic and Microfluidic Devices in Transparent Materials" ed. by R. Osellame, G. Ce- rullo, and R. Ramponi, (Springer, Topics in Applied Optics 123, 2014) p. 315.

[35] G.-L. Roth, S. Hessler, S. Kefer, M. Girschikofsky, C. Esen, and R. Hellmann: Opt Express, 28, (2020) 18077.

[36] B. Horváth, P. Ormos, and L. Kelemen: Micromachines, 8, (2017) 219.

[37] G.-L. Roth, C. Esen, and R. Hellmann: J. Laser Appl., 31, (2019) 22603.

[38] Y. Zhang, G. Cheng, G. Huo, Y. Wang, W. Zhao, C. Mauclair, R. Stoian, and R. Hui: Laser Phys., 19, (2009) 2236.

[39] D. Choudhury, J. R. Macdonald, and A. K. Kar: Laser Photonics Rev., 8, (2014) 827.

[40] F. Chen and V. de Aldana, JR: Laser Photonics Rev., 8, (2014) 251.

[41] M. Rosenberger, S. Hessler, S. Belle, B. Schmauss, and R. Hellmann: Sensors and Actuators A: Physical, 221, (2015) 148.

[42] S. Hessler, M. Rosenberger, B. Schmauss, and R.

Hellmann: Opt. Mater., 75, (2018) 230.

(Received: June 27, 2020, Accepted: February 3, 2021) 